\title{
Obesity and respiratory diseases
}

This article was published in the following Dove Press journal:

International Journal of General Medicine

20 October 2010

Number of times this article has been viewed

\section{Christopher Zammit Helen Liddicoat \\ Ian Moonsie \\ Himender Makker}

Sleep and Ventilation Unit,

Department of Respiratory Medicine, North Middlesex University Hospital, London, UK

Correspondence: Himender K Makker Sleep and Ventilation Unit, Chest Clinic, North Middlesex University Hospital, Sterling Way, London NI8 IQX, UK Email himender.makker@nmh.nhs.uk
Abstract: The obesity epidemic is a global problem, which is set to increase over time. However, the effects of obesity on the respiratory system are often underappreciated. In this review, we will discuss the mechanical effects of obesity on lung physiology and the function of adipose tissue as an endocrine organ producing systemic inflammation and effecting central respiratory control. Obesity plays a key role in the development of obstructive sleep apnea and obesity hypoventilation syndrome. Asthma is more common and often harder to treat in the obese population, and in this study, we review the effects of obesity on airway inflammation and respiratory mechanics. We also discuss the compounding effects of obesity on chronic obstructive pulmonary disease (COPD) and the paradoxical interaction of body mass index and COPD severity. Many practical challenges exist in caring for obese patients, and we highlight the complications faced by patients undergoing surgical procedures, especially given the increased use of bariatric surgery. Ultimately, a greater understanding of the effects of obesity on the respiratory disease and the provision of adequate health care resources is vital in order to care for this increasingly important patient population.

Keywords: obesity, lung function, obstructive sleep apnea, obesity hypoventilation syndrome, anesthesia

\section{Introduction}

Obesity is defined as a body mass index (BMI) greater than $30 \mathrm{~kg} / \mathrm{m}^{2}$. Over 1.6 billion adults worldwide are overweight, of which 400 million are obese. The World Health Organization predicts that $10 \%$ of the global population will be obese by $2015 .{ }^{1}$ The prevalence of obesity in European countries has tripled over the past 2 decades, with significant health and economic burdens. ${ }^{2}$ In the United Kingdom, obesity contributes to 30,000 deaths a year and $£ 3.5$ billion (US $\$ 5.4$ billion) in health care costs. ${ }^{3}$ In the United States of America, the annual health care costs are 36\% greater for an obese patient compared with a patient with a normal BMI. ${ }^{4}$

This increased morbidity and mortality associated with obesity is linked to its role in numerous chronic medical conditions, including cardiovascular and metabolic diseases, hypercoagulable states, lower back pain, osteoarthritis, and cancer. Obesity is also strongly linked with respiratory symptoms and diseases, including exertional dyspnea, obstructive sleep apnea syndrome (OSAS), obesity hypoventilation syndrome (OHS), chronic obstructive pulmonary disease (COPD), asthma, pulmonary embolism, and aspiration pneumonia. ${ }^{5-7}$

Many obese patients have breathlessness on exertion, and the negative physiological effect of obesity on lung function has been demonstrated in numerous studies. 
However, the influence of obesity in respiratory diseases is complex and goes beyond the obvious mechanical and physical consequences of weight gain and its associated inflammatory and metabolic disorders.

\section{Obesity and respiratory mechanics}

Weight gain and rising BMI are associated with decreases in lung volumes, which are reflected by a more restrictive ventilatory pattern on spirometry. Cross-sectional and longitudinal studies have demonstrated that a rise in BMI lowers forced expiratory volume in 1 second (FEV1), forced vital capacity (FVC), functional residual capacity (FRC), and the expiratory reserve volume (ERV). In morbid obesity $\left(\mathrm{BMI}>40 \mathrm{~kg} / \mathrm{m}^{2}\right)$, there is also a modest decrease in residual volume (RV) and total lung capacity (TLC). At such extreme levels of obesity, FRC approaches RV. ${ }^{4,8-12}$

\section{Central and peripheral obesity}

Two distinct patterns of obesity are recognized in the general population: central and peripheral obesity. Central obesity is more common in males, with increased adipose tissue in the anterior chest wall, anterior abdominal wall, and visceral organs. Peripheral obesity is more common in women than men, with adiposity located peripherally in the subcutaneous tissue. $^{4}$

Abdominal obesity is associated with worsening lung function and respiratory symptoms. ${ }^{12}$ Studies that focused on waist circumference (WC) found that elevated waist-tohip ratio and abdominal height had good correlation with impaired lung function. ${ }^{12-14}$ Various medical conditions are also associated with an elevated WC. These include cardiovascular diseases (atherosclerosis, ischemic heart disease, stroke, hyperlipidemia, and hypertension), type 2 diabetes mellitus, and the metabolic syndrome. ${ }^{11,13}$ Peripheral obesity is associated with fewer medical complications and better lung function. ${ }^{4}$

Excess weight on the anterior chest wall due to obesity lowers chest wall compliance and respiratory muscle endurance with increase in work of breathing and airway resistance. ${ }^{4,11,15}$ Furthermore, the buildup of adipose tissue in the anterior abdominal wall and in the intra-abdominal visceral tissue hinders diaphragmatic movement, diminishes basal lung expansion during inspiration, and with the closure of peripheral lung units, causes ventilation-perfusion abnormalities and arterial hypoxemia. ${ }^{12,16}$ These changes contribute to an increase in prevalence of respiratory problems in obese individuals, particularly on exertion and in the supine position such as during sleep and perioperatively during anesthesia.
Two recent studies have demonstrated that changes in lung volume can occur at early stages of obesity and are not limited to the morbidly obese individuals (BMI $\left.>40 \mathrm{~kg} / \mathrm{m}^{2}\right) .{ }^{10,17}$ Reduction in FRC and ERV due to loss of basal lung volume can occur even in overweight patients $\left(\mathrm{BMI}>25-30 \mathrm{~kg} / \mathrm{m}^{2}\right.$ ) or relatively lean individuals with a $\mathrm{WC}$ above the normal range ( $>35$ in in women; $>40$ in in men). The site of fat accumulation is crucial in determining the effect of obesity on respiratory system mechanics. BMI alone does not provide sufficient information about the bodily distribution of fat mass (FM). ${ }^{12,13}$

\section{Pulmonary function and the metabolic effects of obesity}

The relation between FEV1 and BMI is not a straightforward one; FEV1 drops at a much lower rate than FVC or ERV. The association between an increase in BMI and reduction in FVC is stronger than with FEV1. Obesity is more often associated with a restrictive lung ventilatory defect than an obstructive one, with a low FVC and an elevated FEV1/FVC ratio $(>70) \cdot{ }^{9,12,13}$

Low FEV1 values have also been observed in other chronic medical diseases, such as hypertension, dyslipidemia, cerebrovascular diseases, and lung cancer. This association with chronic medical conditions is not clear. However, it appears that FEV1 is a good predictor of all-cause morbidity and mortality, with increased $\mathrm{C}$-reactive protein (CRP) values correlating with reductions in FEV1.4,13,18,19

Abdominal obesity and poor lung function are associated with a low-grade inflammatory state, which might contribute to metabolic disease and ill-health. Elevated levels of interleukins (ILs) 6 and 8, tumor necrosis factor $\alpha$ (TNF- $\alpha$ ), CRP, leptin, and lower levels of adiponectin, which helps in regulating insulin sensitivity, have all been observed. Adipose tissue is now regarded as an endocrine organ, with release of adipocytokines affecting systemic inflammation possibly triggered by hypoxemia induced by obesity and related respiratory disorders like OSA, OHS, or COPD. ${ }^{13,18}$

In a study by Leone et al ${ }^{13}$ impaired lung function (FEV1, FVC, FEV1/FVC) was associated with the components of the metabolic syndrome: most strongly with abdominal obesity (higher WC) and with elevated lowdensity lipoproteins, hypertension, and insulin resistance. These correlations were independent of age, sex, BMI, history of cardiovascular diseases, smoking, or alcohol use. All reduced spirometric measures were associated with a restrictive lung pattern, typically seen in obesity-related lung changes. ${ }^{13}$ 


\section{Obesity and respiratory symptoms}

Overweight and obese individuals are more likely to have respiratory symptoms than individuals with a normal BMI, even in the absence of demonstrable lung disease. Studies have shown an increase in self-reported dyspnea and wheezing at rest and on exertion in obese compared with lean individuals..$^{14,15,20}$ In a large epidemiological survey of Swedish obese patients awaiting bariatric surgery, $80 \%$ reported dyspnea on exertion of two flights of stairs. ${ }^{14}$ Recently, Babb et $\mathrm{al}^{15}$ reported a heightened sensation of breathlessness on exertion in $37 \%$ of 30 otherwise healthy obese women.

Kress et $\mathrm{al}^{21}$ calculated respiratory muscle oxygen consumption during ventilation $\left(\mathrm{VO}_{2}\right.$ in $\left.\mathrm{L} / \mathrm{min}\right)$ in a group of intubated and anesthetized morbidly obese patients compared with lean controls. Obese subjects exhibited a $16 \%$ increase in respiratory work and oxygen demand. ${ }^{21}$ In a study of healthy obese women (BMI $\left.>30 \mathrm{~kg} / \mathrm{m}^{2}\right)$, Babb et al ${ }^{15}$ also linked exertional dyspnea with an elevated work of breathing. They observed a $70 \%$ increase in oxygen demand in dyspneic obese women when compared with obese controls with significantly less exertional breathlessness. Interestingly, there was no other identifiable difference in pulmonary function, respiratory system mechanics, body composition, or fat distribution in the study. Furthermore, the authors found no evidence to support cardiac deconditioning in these patients; all the subjects had a normal measured peak $\mathrm{VO}_{2}(\mathrm{~L} / \mathrm{min}) .{ }^{15}$ The above studies suggest that the sensation of increased dyspnea in obesity is related to an increased oxygen demand during ventilation, which is aggravated further by exertion.

Respiratory muscle function has been shown to deteriorate in obesity, in a pattern similar to that seen in chronic respiratory disease like COPD. This may also be related to a reduction in the overall fat free mass (FFM - ie, muscle mass). ${ }^{18,22}$ This deficiency may contribute to the additional oxygen demand required for ventilation and may heighten sensation of breathlessness in obese patients. In addition, studies have demonstrated that weight loss helps restore the function of respiratory muscle. Therefore, the elevated mechanical work load of obesity can overburden respiratory muscles through a combination of increased work of breathing and apparent reduction in respiratory muscle efficiency. ${ }^{14,22}$

Finally, El-Gamal et $a^{20}$ investigated the increased respiratory drive commonly found in normocapnic obese individuals and its association with increasing breathlessness and lower lung volumes. Both respiratory symptoms and functions improved following bariatric surgery. This further emphasizes the fact that weight loss can reverse the reduction in pulmonary function and increased dyspnea commonly associated with obesity. ${ }^{20}$

It is still unclear why only a proportion of obese individuals experience shortness of breath on exertion. Frequently, coexisting medical conditions exist that can act as confounding factors when attempting to assess the specific contribution obesity plays in the development of respiratory symptoms. Some of the most common include smoking, COPD, and cardiovascular diseases. ${ }^{14}$

\section{Obesity and OSA}

OSA is characterized by repeated obstructive apneas due to a collapsible upper airway (UA) while asleep. This results in repetitive nocturnal oxygen desaturations, fragmented sleep, and excessive daytime somnolence. ${ }^{23-25}$ The condition is treated by application of continuous positive airways pressure (CPAP) via a face or nasal mask while the subject is asleep. With an "obesity epidemic" currently underway, the incidence and prevalence of OSA have now reached new heights and is predicted to rise even further. ${ }^{25}$

\section{Pathophysiology of OSA}

The risk of developing OSA increases considerably at a higher BMI. If morbidly obese (BMI $>40 \mathrm{~kg} / \mathrm{m}^{2}$ ), the risk of an individual of developing OSA lies between 55\% and 90\%. ${ }^{26}$ Other contributing factors include a large neck circumference ( $>17$ in) and a higher oral mallampati score (denoting an anatomically narrow UA). Neck circumference is a better predictor of OSA than BMI. Even more interestingly, abdominal circumference has proven to be a better predictor of OSA than both neck circumference and BMI. ${ }^{27-29}$

In OSA, the UA collapses at a critical closing pressure $\left(\mathrm{P}_{\text {crit }}\right)$. It is believed that disturbances in the neuromuscular control of the pharyngeal dilatory muscles fail to protect the UA against increasing extra-luminal pressures. ${ }^{30}$ The craniofacial variations can predispose toward an anatomically unfavorable UA. Obesity seems to play a large part in increasing the passive mechanical pressures, which contribute to UA obstruction by increasing fat deposition around the soft tissues of the neck and tongue, contributing to an increase in extra-luminal pressures in the pharynx that elevates $\mathrm{P}_{\text {crit }}$ thereby increasing the chances of airway collapse. ${ }^{23}$ Elevated levels of obesity have also been shown to worsen nocturnal hypoxemia in OSA, in part due to an increase in oxygen demand. During apnea, obese patients desaturate at a faster level than lean controls. ${ }^{31}$

Obesity-related reductions in lung volumes (ie, FRC and TLC) also increase passive closing pressures at the pharynx. ${ }^{32}$ 
Heinzer et $\mathrm{al}^{33}$ demonstrated that a $1.3 \mathrm{~L}$ increase in FRC during sleep decreased the apnea/hypopnea index (AHI) from 62.3 events per hour to 31.2 events per hour. In an earlier study by the same authors, higher CPAP pressures were found to be required in order to overcome UA obstruction at reduced lung volumes. ${ }^{34}$ It appears that lower lung volumes decrease the caudal traction on the UA during lung inflation, producing lesser longitudinal tension on the pharyngeal walls, especially in the supine position. ${ }^{35}$

Obesity is known to account for approximately 30\%-50\% of the variability in AHI. ${ }^{24,25}$ Numerous longitudinal studies have demonstrated that increasing weight aggravates the AHI, which defines the severity of OSA. There is still some debate as to whether this association is gender-related. An increase in body weight was observed to have a greater effect on sleep apnea in men and postmenopausal women compared with premenopausal women; however, the data were not consistent among the studies. ${ }^{36,37}$ It was also noticed that the rate of increase in apneas is exponential, with steeper elevations in AHI at higher levels of BMI. Thus, an advice to avoid gaining further weight seems as important as to that of losing body weight. ${ }^{36}$ Regrettably, the converse does not seem to apply. For the same reductions in body weight, AHI decreases at a much lower rate. ${ }^{36,37}$ Weight loss can be difficult to achieve and even harder to maintain. A viscous cycle often develops in patients with OSA, where lack of good quality sleep results in lower energy levels during the daytime, with a subsequent decline in physical activity. This reduction in exercise promotes further weight gain. In addition, the increasing body weight worsens fatigability and exacerbates the OSA.

There are evidence to suggest that poor sleep exacerbates weight gain by a direct effect on food intake. In a study on healthy subjects who had their sleep time restricted to less than 4 hours per night for two nights, an increased intake of carbohydrates and calories-dense foods was reported. In addition, they were observed to have lower serum leptin and elevated ghrelin levels, where leptin is known to act as an appetite suppressant, whereas ghrelin seems to stimulate hunger. ${ }^{25}$

A number of studies have focused on diet-induced weight loss, achieving promising results in patients who underwent intensive weight management programs with moderate reductions in overall weight and AHI. Of note, Toumilehto et al ${ }^{38}$ achieved a $63 \%$ remission rate in 72 adults with mild OSA subjected to very low calorie feeds. However, motivation is of paramount importance in order to attain significant results, and a few studies exist on the long-term sustained benefit of such programs.
Lettieri et $\mathrm{al}^{26}$ studied the improvement in AHI in 24-selected OSA patients with morbid obesity who underwent bariatric surgery. At 1 year follow-up, despite a mean BMI reduction of $18.4 \mathrm{~kg} / \mathrm{m}^{2}$ and an average drop in AHI of 23.4 events per hour, only one patient experienced complete resolution of OSA. The remaining patients required continual use of CPAP, albeit at lower pressure. In the study, OSA severity reduced by $50 \%$. However, two patients were found to have an increase in their AHI despite significant weight loss. ${ }^{26} \mathrm{~A}$ meta-analysis by Greenburg et a ${ }^{39}$ representing 342 patients in the year 2009, showed similar results with bariatric surgery significantly reducing AHI, however, to a mean value still consistent with moderately severe OSA. ${ }^{39}$

Moderate OSA (AHI > 15 events per hour) and severe OSA (AHI > 30 events per hour) are associated with an increased mortality. Reducing disease severity to an AHI of $<15$ events per hour (ie, mild OSA) through weight loss can help in achieving improved outcomes in OSA patients. ${ }^{40}$ A continual effort to lose weight could also help in preventing the emergence of associated medical conditions like insulin resistance, diabetes, and cardiovascular diseases.

\section{Obesity hypoventilation syndrome}

Obesity plays a key role in the pathogenesis of OHS. A BMI of more than $30 \mathrm{~kg} / \mathrm{m}^{2}$ (ie, clinical obesity) together with daytime hypercapnia $\left(\mathrm{PaCO}_{2}>6 \mathrm{kPa}\right)$ is required for the diagnosis of OHS.

Patients with OHS share many physical similarities with patients with OSA, including obesity, higher mallampati scores, and elevated neck circumference and WC. Many OHS patients suffer from sleep-disordered breathing (SDB) and commonly present with a history of snoring and witnessed apneas. The exact prevalence of OHS in the general population is still unknown although epidemiological studies seem to suggest that this might be around $10 \%$ of OSA patients. The largest study $(n=1,141)$ examining the prevalence of hypercapnia in OSA patients discovered an elevated daytime hypercapnia in $11 \%$ of patients. On evaluating the relationship between the increased $\mathrm{PaCO}_{2}$ and severity of obesity, they found the prevalence to be higher with rising BMI. ${ }^{41}$

However, $\mathrm{CO}_{2}$ retention in OHS seems to be subject to a multitude of factors other than an elevated BMI. Indeed, many obese patients who develop OSA do not progress to OHS and hypercapnia. In addition, a small proportion of OHS patients never develop SDB and OSA but remain as "pure" OHS patients exhibiting chronic hypoventilation and daytime hypercapnea. ${ }^{42}$ It is believed that normocapnic obese subjects manage to overcome the mechanical constraints of 
obesity-related restrictive lung disease by augmenting their respiratory drive and minute ventilation to maintain a normal $\mathrm{PaO}_{2}$ and $\mathrm{PaCO}_{2}$. However, in OHS patients, this respiratory compensation is lost by disturbances in their central control of respiration. ${ }^{42}$

Many studies have focused on the effects of central adipose tissue, whose metabolic and endocrine functions have been speculated to influence the central control of breathing. ${ }^{20,42,43}$ Of note, leptin is an adipose-derived hormone that helps in preventing excessive food intake by suppressing appetite, thereby helping to prevent additional weight gain. It has been proposed that leptin may also play a role in maintaining an adequate minute ventilation in obese subjects. Leptin-deficient obese mice were observed to have a reduced ventilatory drive in response to hypercapnia, which subsequently improved on administration of leptin. ${ }^{43}$

A significant proportion of obese patients have elevated leptin levels, suggesting a build up of tolerance toward its effects (ie, leptin resistance). Makinodan et $\mathrm{al}^{44}$ demonstrated a significant association between hypercapnic ventilatory response and higher serum leptin levels in obese patients with or without OSA. Furthermore, in OSA with chronic hypercapnia, the leptin levels were even higher. ${ }^{44}$ Finally, Chin et $\mathrm{al}^{45}$ demonstrated a noticeable reduction in leptin levels following CPAP treatment. ${ }^{45}$ All these studies propose an important role of leptin and highlight the potential significance of the development of leptin resistance in obesity and OHS.

It is important to distinguish among OSA, OHS, and OSA/OHS overlap as treatments can be different. These conditions could be termed as obesity-related respiratory failure. Noninvasive ventilation is used to treat patients with hypercapnic respiratory failure due to OHS and patients who fail to have adequate ventilation with CPAP. In some centers, OHS is among the most frequent indications for domiciliary ventilation. ${ }^{46}$ However, this practice is supported mainly by retrospective and case-control analyses, which have shown improvement in physiological and health-related quality of life parameters. More prospective, randomized, and controlled trials are needed to evidence the management of this growing group of patients.

OHS patients often have higher BMIs, more severe daytime hypercapnia, and more elevated AHI levels than OSA patients. Nocturnal oxygen desaturation events are often more profound and frequent, resulting in deeper levels of nocturnal hypoxemia and hypercapnia. This can predispose to a higher sympathetic activation, elevated oxidative stress, and higher cardiovascular risk factors.
This is compounded by related disorders of the metabolic syndrome (ie, hypercholesterolemia, insulin resistance, and diabetes). Chronic daytime hypoxemia and hypercapnia in OHS patients is associated with a high risk of developing pulmonary hypertension, right-sided heart failure, and cor pulmonale. Not surprisingly, the mortality and morbidity from OHS have been found to be much higher than from both OSA and obesity alone. ${ }^{42}$

\section{Obesity and asthma}

Cross-sectional and longitudinal studies have linked obesity with asthma. The frequency of self-reported symptoms of breathlessness and wheezing increases with BMI in patients with asthma. Furthermore, asthma seems to be commoner in the overweight and obese population. In a meta-analysis of epidemiological studies, Beuther and Sutherland ${ }^{47}$ demonstrated that the prevalence of asthma was higher by $38 \%$ in overweight patients and by $92 \%$ in obese patients. They found no differences in the incidence between genders. ${ }^{47}$ Another study by Aaron $\mathrm{SD}$ et $\mathrm{al}^{49}$ looked at the possible over-diagnosis of asthma in obese patients; however, despite a higher rate of misdiagnosis of asthma in all patients (up to one-third), there was no clear association with obesity.

Studies have not ascertained whether increased respiratory symptoms are attributable to asthma or being overweight. Schachter et $\mathrm{al}^{48}$ demonstrated that the symptoms of asthma increased with a rise in BMI; however, this was not associated with an increase in airway hyper-responsiveness (AHR) ${ }^{49}$ McLachlan et $\mathrm{al}^{50}$ found that although inhaled nitric oxide was increased in patients with asthma, there was no association with either BMI or percentage body fat. Similarly, obese patients who manage weight loss by diet or bariatric surgery are found to have improvements in their lung function and asthma symptoms. However, there are no changes in AHR or inhaled nitric oxide. ${ }^{51,52}$ BMI does not seem to affect eosinophil counts or serum IgE levels either. ${ }^{53}$

Without demonstrable correlation between obesity and airway inflammation, it has been, therefore, speculated that the epidemiological association between obesity and asthma is primarily due to ventilatory mechanics rather than an inflammatory one. ${ }^{54}$ King et $\mathrm{al}^{55}$ observed a significant decrease in airway caliber with increasing weight but observed no change in AHR or asthma rates. Weight gain causes volume loss and a decrease in lung compliance with reduction in airway size and tone. These changes can increase airflow resistance and obstruction, with reduction in airway conductivity. ${ }^{4,10}$ Reduced airway caliber may predispose toward airway trapping and dynamic hyperinflation leading 
to increasing dyspnea and wheezing, particularly during respiratory tract infection.

It has also been suggested that systemic inflammation associated with obesity may contribute to glucocorticoid insensitivity observed in a few subjects with asthma. Obese subjects have elevated inflammatory mediators, namely IL-6, IL-8, and TNF- $\alpha$, which potentially could affect glucocorticoid sensitivity. ${ }^{56}$ Elevated leptin levels in obesity have also been implicated; administered leptin in mice promoted AHR and increased IgE levels. ${ }^{57}$ Finally, Peters-Golden et $\mathrm{al}^{58}$ demonstrated that the clinical response to beclomethasone declined with increasing BMI.

Obese patients with asthma are known to suffer from more acute attacks, increased use of asthma medication, frequent visits to the emergency department (ED), and more hospital admissions than nonobese patients with asthma. In a prospective cohort study, Rodrigo and Plaza ${ }^{54}$ looked at adult patients who presented to the ED for the treatment of acute asthma. They observed that overweight and obese patients had a longer duration of asthmatic symptoms and a higher rate of use of inhaled corticosteroids and theophylline than normal body weight patients with asthma. Interestingly, despite more severe dyspnea and wheezing, in patients with asthma with a BMI $>25 \mathrm{~kg} / \mathrm{m}^{2}$, they had a higher initial FEV1 and peak expiratory flow than nonobese patients with asthma. However, obese patients with asthma required a longer stay at the ED for treatment, a lower discharge rate from the ED, and a higher hospitalization rate than nonobese patients with asthma. ${ }^{54}$

Although there is a paucity of evidence linking obesity, AHR, and airway inflammation, the overlap in the respiratory symptoms of asthma and obesity patients cannot be ignored. Clinicians need to be cautious when diagnosing asthma in these patients. Appropriate investigations are often necessary to establish the diagnosis and to help rule other possible coexisting medical conditions contributing to the symptoms of dyspnea and wheezing.

\section{Obesity and COPD}

COPD is characterized by the progressive and largely irreversible airflow obstruction and occurs predominately in smokers. ${ }^{59}$ COPD and obesity share a complex interplay of similarities, which seems to compound each condition. Both are associated with deterioration in lung function, hypoxia, and a low-grade systemic inflammation, which predispose to increasing medical morbidity and mortality.

The combined effects of obesity and COPD add to deterioration of lung function. FEV1 declines as both conditions worsen. ${ }^{4,11}$ COPD worsens airflow obstruction and hypoxia and, in addition to smoking, increases pulmonary and systemic oxidative stress. This is particularly true in the overlap syndrome where the combination of COPD, obesity, and OSA worsens nocturnal and daytime hypoxemia and hypercapnia, with over activation of the sympathetic system leading to increased cardiovascular and metabolic morbidity and mortality with evidence of increased local and systemic inflammation. ${ }^{60}$ Furthermore, in patients with OSA and COPD (overlap syndrome), there is a heightened risk of developing respiratory failure, pulmonary hypertension, and cor pulmonale compared with patients with only COPD. This is independent of the degree of airflow obstruction. ${ }^{56}$

Studies have demonstrated that in early COPD (GOLD stages 1 and 2), obesity correlates positively with a rise in mortality. This association is strongest with elevated FM and increased WC rather than BMI or FFM. It is purported that as a consequence of increasing exertional dyspnea secondary to COPD, there is a reduction in physical exercise. The use of oral steroid medication also increases; patients put on more weight and FM and undergo skeletal muscle deconditioning with loss of FFM. ${ }^{18}$

There is a higher prevalence of overweight and obesity in the early COPD population compared with the general population, but a paradoxically lower BMI in moderate to severe COPD patients (GOLD stages 3 and 4). ${ }^{61,62}$ At these later stages of COPD, lower values for BMI $\left(<25 \mathrm{~kg} / \mathrm{m}^{2}\right)$ seems to adversely affect prognosis. The strongest correlation seems to occur with deterioration in FFM, often associated with an overall decline in BMI and decrease in FM. In COPD and obesity, low-grade inflammation and arterial hypoxemia have been associated with a reduction in skeletal muscle tissue, a decrease in muscle fat oxidative capacity, a shift from muscle fiber type 1 (slow twitch) to type 2 (fast twitch), and a loss of respiratory muscle performance. ${ }^{18}$

Physiological and metabolic factors related to COPD and obesity seems to jeopardize morbidity and mortality further when in association. It is clear that these interactions are complex and additional studies are required in order to further improve our understanding on both conditions.

\section{Obesity and anesthesia}

With an ever-increasing demand for surgical procedures involving anesthesia coupled with a mounting global over-weight population, it is little wonder that obesity has become such a common and important factor in routine anesthetic assessment. Obesity and its related disorders present an anesthetist with numerous difficulties necessitating the need for careful 
planning and awareness of the common associated pitfalls. In particular, OSA and OHS should always be considered in the obese patient undergoing assessment for surgery. ${ }^{27}$

Obesity can contribute to complications at all stages of anesthesia. It makes intubation and ventilation difficult because of an increase in neck size and small UAs. Anatomical landmarks are often less straightforward, with frequent difficulties in establishing vascular access and catheterization. Increasing weight makes ambulation difficult and appropriate positioning burdensome for radiographic imaging. Drug pharmacokinetics is often complex due to a disproportionate amount of adipose tissue. Obese surgical patients face higher anesthetic risks and often require a longer hospital stay with higher health care costs. They are prone to more postoperative cardiac and pulmonary complications including pneumonia (associated with a 30\%-46\% mortality rate) and thromboembolic disease. ${ }^{13,63}$

\section{Intubation and airway management}

Obese patients often have a thicker neck, smaller UA caliber, and poor neck mobility. Mallampati score, used to predict difficulties in intubation by gauging tongue size within the limited oral cavity, is often high in obese patients and, particularly, in obese OSA patients. Langeron et a ${ }^{64}$ identified patients $>55$ years of age, BMI $>26 \mathrm{~kg} / \mathrm{m}^{2}$, snoring, beard, and lack of teeth as independent risk factors for difficult mask ventilation. Similarly, Kheterpal et a ${ }^{65}$ listed limited mandibular protrusion, a thick neck, sleep apnea, snoring, and BMI $>30 \mathrm{~kg} / \mathrm{m}^{2}$ as predictors of a complicated intubation. ${ }^{65}$

OSA and OHS patients often have uncoordinated UA dilatory muscle activity, which causes additional problems in maintaining an airway. ${ }^{30}$ This is exacerbated further with sedative and anesthetic drugs required during procedures, whose dosage can be difficult to determine due to increased volume of distribution. Smaller lung volumes have also been shown to increase the chances of UA collapsibility. ${ }^{32,33}$

All this is compounded by the fact that the anesthetist needs to successfully intubate within a smaller time window as obese patients tend to desaturate faster. ${ }^{16,31}$ Lower FRC and ERV means that obese patients function at reduced oxygen reserves. An increased work in breathing places additional strain on already impaired cardiac and pulmonary functions. Coexisting medical diseases related to obesity further complicate anesthesia. Particularly, OSA and OHS patients have higher risks for systemic and pulmonary hypertensive diseases, coronary artery diseases, cardiac arrhythmias, congestive cardiac failure, and diabetes. Gastroesophageal reflux disease can predispose to regurgitation and aspiration pneumonia. ${ }^{16}$

\section{Ventilation and atelectasis}

In the anesthetized patient, mechanical ventillation mimics normal breathing but does not reproduce its physiological effects. Mechanically ventilated patients develop pulmonary atelectasis, which leads to hypoxemia by increased ventilation-perfusion mismatch and venous shunting. Atelectatic lung decreases the FRC; collapsed lung contains 4 times more lung tissue by volume than aerated lung. This is more problematic in the obese patient, as lower lung volumes and diminished airway calibers predispose to compression atelectasis. In the supine position, this is most commonly seen in the posterior-dependent regions of the lung, close to the diaphragm. Mechanical ventilation distorts normal diaphragmatic movement, which is aggravated by the increased abdominal pressure present with central adiposity ${ }^{63}$ Lung atelectasis has been shown to resolve within 24 hours in nonobese patients following laparoscopy and within 2 days after major surgery. With morbid obesity, lung atelectasis persists for much longer, increasing the risk of the postoperative patient to recurrent hypoxemia and postoperative pulmonary complications. ${ }^{66}$

\section{Extubation and postoperative recovery}

Obesity also poses serious threats during extubation and in the immediate postoperative period during the transfer and observation of the patient in the recovery room. Ambulation of the patient can make continuous monitoring of vital statistics difficult to achieve. OSA and OHS patients inherently have a smaller UA, uncoordinated pharyngeal muscle activity, and a blunted chemoreceptive control of their ventilation. All these factors are made worse by the residual effects of administered anesthetic drugs and postextubation pharyngeal edema. Therefore, extubation can leave a postoperative patient with recurrent UA obstruction and hypoxemia.

Regional anesthesia like spinal or epidural anesthesia may offer advantages in the obese surgical patient although not without its own technical difficulties. An unsuccessful block is 1.6 times more likely in the obese patient. ${ }^{29}$ Conscious sedation is also useful, avoiding deep sedation by careful titration. ${ }^{27}$ If, however, general anesthetic is required, rapid sequence induction is often advocated. Application of a higher positive end-expiratory pressure (PEEP) than usual (PEEP $10 \mathrm{~cm}$ of water) in the morbidly obese patient is effective for the prevention of atelectasis during induction and when atelectasis occurs intraoperatively. ${ }^{63}$ Awake intubation should be considered if serious difficulties are anticipated. 
During anesthetic recovery, high-flow oxygen administered in the sitting or lateral position is fundamental for the obese patient. Continuous or bilevel positive airway pressure (CPAP or BiPAP) administered via face mask can help in preventing UA obstruction and basal lung atelectasis immediately following extubation. This is especially true in OSA or OHS patients. ${ }^{67}$ Joris et $\mathrm{al}^{67}$ examined the effect of nasal BiPAP in obese patients given for the first 24 hours after undergoing gastroplasty. BiPAP with an inspiratory and expiratory positive airway pressure of 12 and $4 \mathrm{~cm} \mathrm{H}_{2} \mathrm{O}$, respectively (12/4), significantly reduced the fall in FEV1, FVC, and oxygen saturation from preoperative baseline, when compared with a lower BiPAP pressure of $8 / 4 \mathrm{~cm} \mathrm{H}_{2} \mathrm{O}$ or only supplemental face mask oxygen. Pulmonary function also recovered more rapidly in the higher pressure BiPAP group. ${ }^{47}$

Strategies may be applied before, during, and after anesthesia of the obese patient to reduce perioperative complications. Untreated OSA patients should be introduced to CPAP therapy before elective surgery and patients are often encouraged to bring their own machine with them for the operation. Following extubation, these patients struggle less against an administered CPAP mask. In addition, studies have shown that commencing CPAP therapy at least a week before surgery helps improve UA collapsibility, reduce leptin levels, and augment respiratory drive and chemoresponsiveness. ${ }^{27}$

\section{Conclusion}

Obesity presents with various respiratory problems and is associated with many medical comorbidities. Despite the evidence demonstrating additional health risks and reduced quality of life, the rate of obesity is still increasing globally. Although genetic susceptibility does play a part, much can be done in treatment and prevention of obesity to reduce and minimize many medical and respiratory complications. Governments need to continue working together with public health to encourage a lifestyle with improved diet and increased physical activity.

\section{Disclosure}

The authors report no conflicts of interest in this work.

\section{References}

1. World Health Organization Obesity and overweight. Fact Sheet No 311. Available from: http://www.who.int/mediacentre/factsheets/fs311/en/ index.html. Updated Sep 2006. Accessed 2010 May 15.

2. Lean M, Lara J, Hill JO. ABC of obesity. Strategies for preventing obesity. BMJ. 2006;333:959-962.

3. Haslam D, Sattar N, Lean M. ABC of obesity. Obesity - time to wake up. BMJ. 2006;333:640-642.

4. McClean KM, Kee F, Young IS, Elborn JS. Obesity and the lung: 1. Epidemiology. Thorax. 2008;63:649-654.
5. Koenig SM. Pulmonary complications of obesity. Am J Med Sci. 2001; 321:249-279.

6. Murugan AT, Sharma G. Obesity and respiratory disease. Chron Respir Dis. 2008;5:233-242.

7. Stunkard AJ. Current views on obesity. Am J Med. 1996;100: 230-236.

8. McClean KM, Cardwell CR, Kee F. Longitudinal change in BMI and lung function in middle-aged men in Northern Ireland. Ir J Med Sci. 2007;176 Suppl 10:S418.

9. Nakajima K, KubouchiY, Muneyuki T, Ebata M, Eguchi S, Munakata H. A possible association between suspected restrictive pattern as assessed by ordinary pulmonary function test and the metabolic syndrome. Chest. 2008;134:712-718.

10. Jones RL, Nzekwu MU. The effects of body mass index on lung volumes. Chest. 2006;130:827-833.

11. Ochs-Balcom HM, Grant BJ, Muti P, et al. Pulmonary function and abdominal adiposity in the general population. Chest. 2006;129: $853-862$.

12. Wannamethee SG, Shaper AG, Whincup PH. Body fat distribution, body composition, and respiratory function in elderly men. Am J Clin Nutr. 2005;82:996-1003.

13. Leone N, Courbon D, Thomas F, et al. Lung function impairment and metabolic syndrome. The critical role of abdominal obesity. Am J Respir Crit Care Med. 2009;179:509-516.

14. Gibson GJ. Obesity, respiratory function and breathlessness. Thorax. 2000;55:S41-S44.

15. Babb TG, Ranasinghe KG, Comeau LA, Semon TL, Schwartz B. Dyspnoea on exertion in obese women. Am J Respir Crit Care Med. 2008;178:116-123.

16. Malhotra A, Hillman D. Obesity and the lung: 3. Obesity, respiration and intensive care. Thorax. 2008;63:925-931.

17. Babb TG, Wyrick BL, deLorey DS, Chase PJ, Young MY. Fat distribution and end-expiratory lung volume in lean and obese men and women. Chest. 2008;134:704-711.

18. Franssen FME, O'Donnell DE, Blaak EE, Schols AMWJ. Obesity and the lung: 5. Obesity and COPD. Thorax. 2008;63:1110-1117.

19. Hacken Nick HT. Physical inactivity and obesity. Proc Am Thorac Soc. 2009;6:663-667.

20. El-Gamal H, Khayat A, Shikora S, Unterborn JN. Relationship of dyspnoea to respiratory drive and pulmonary function tests in obese patients before and after weight loss. Chest. 2005;128:3870-3874.

21. Kress JP, Pohlman AS, Alverdy J, Hall JB. The impact of morbid obesity on oxygen cost of breathing at rest. Am J Resp Crit Care Med. 1999; 160:883-886.

22. Laghi F, Tobin MJ. Disorders of the respiratory muscles. Am J Respir Crit Care Med. 2008;168:10-48.

23. Patil SP, Schneider H, Marx JJ, Gladmon E, Schwartz AR, Smith PL. Neuromechanical control of upper airway patency during sleep. J Appl Physiol. 2007;102:547-556.

24. Mokhlesi, Gozal. Update on sleep medicine 2009. Am J Respir Crit Care Med. 2010;181:545-549.

25. Crummy F, Piper AJ, Naughton MT. Obesity and the lung: 2. Obesity and sleep-disordered breathing. Thorax. 2008;63:738-746.

26. Lettieri CJ, Eliasson AH, Greenburg DL. Persistence of obstructive sleep apnea after surgical weight loss. J Clin Sleep Med. 2008;4(4): 333-338.

27. Isono S. Obstructive sleep apnea of obese adults. Anesthesiology. 2009; 110:908-921.

28. Scafer H, Pauleit D, Sudhop T, Gouni-Berthold I, Ewig S, Berthold HK. Body fat distribution, serum leptin and cardiovascular risk factors in men with obstructive sleep apnea. Chest. 2002;122:829-839.

29. Candiotti K, Sharma S, Shankar R. Obesity, obstructive sleep apnea, and diabetes mellitus: anaesthetic implications. Br J Anaesth. 2009; 103 Suppl 1:i23-i30.

30. Horner RL. Contributions of passive mechanical loads and active neuromuscular compensation to upper airway collapsibility during sleep. J Appl Phsiol. 2007;102:510-512. 
31. Sato M, Suzuki M, Suzuki J, et al. Overweight patients with severe sleep apnea experience deeper oxygen desaturation at apnoeic events. J Med Dent Sci. 2008;55:43-47.

32. Tagaito Y, Isono S, Remmers JE, Tanaka A, Nishino T. Lung volume and collapsibility of the passive pharynx in patients with sleep-disordered breathing. J Appl Phsiol. 2007;103:1379-1385.

33. Heinzer R, White DP, Malhotra A, et al. Effect of increased lung volume on sleep disordered breathing in patients with sleep apnea. Thorax. 2006;61:435-439.

34. Heinzer RC, Stanchina ML, Malhotra A, et al. Lung volume and continuous airway pressure requirements in obstructive sleep apnea. Am J Resp Crit Care Med. 2005;172:114-117.

35. Peppard PE, Ward NR, Morrell MJ. The impact of obesity on oxygen desaturation during sleep-disordered breathing. Am J Respir Crit Care Med. 2009;180:788-793.

36. Newman AB, Foster G, Givelber R, Nieto FJ, Redline S, Young T. Progression and regression of sleep-disordered breathing with changes in weight. Arch Intern Med. 2005;165:2408-2413.

37. Young T, Peppard PE, Taheri S. Excess weight and sleep-disordered breathing. J Appl Physiol. 2005;99:1592-1599.

38. Toumilehto H, Seppa JM, Markku MP, et al. Lifestyle intervention with weight reduction: first-line treatment in mild obstructive sleep apnea. Am J Respir Crit Care Med. 2009;179:320-327.

39. Greenburg DL, Lettieri CJ, Eliasson AH. Effects of surgical weight loss on measures of obstructive sleep apnea: a meta-analysis. Am J Med. 2009; 122:535-542.

40. Marshall NS, Grunstein RR. Losing weight in moderate to severe obstructive sleep apnea. Editorials. BMJ. 2009;339:b4363.

41. Laaban JP, Chailleux E. Daytime hypercapnia in adult patients with obstructive sleep apnea syndrome in France, before initiating nocturnal nasal continuous positive airway pressure therapy. Chest. 2005;127: 710-715.

42. Teichtahl H. The obesity-hypoventilation syndrome revisited. Chest. 2001;120:336-339.

43. O’Donnell CP, Schaub CD, Haines AS, et al. Leptin prevents respiratory depression in obesity. Am J Respir Crit Care Med. 1999;159: 1477-1484.

44. Makinodan K, Yoshikawa M, Fukuoka A, et al. Effect of serum leptin levels on hypercapnoeic ventilatory response in obstructive sleep apnea. Respiration. 2008;75:257-264.

45. Chin K, Shimizu K, Nakamura T, et al. Changes in intra-abdominal visceral fat and serum leptin levels in patients with obstructive sleep apnea syndrome following nasal continuous positive airway pressure therapy. Circulation. 1999;100:706-712.

46. Chiner E, Llompart M, Martinez-Garcia MA, Fernandez-Fabrellas E, Navarro R, Cervera A. Noninvasive mechanical ventilation in Valencia, Spain: from theory to practise. Arch Bronconeumol. 2009;45(3): 118-122.

47. Beuther DA, Sutherland ER. Overweight, obesity and incident asthma: a meta-analysis of prospective epidemiologic studies. Am J Respir Crit Care Med. 2007;175:661-666.

48. Schachter LM, Salome CM, Peat JK, Woolcock AJ. Obesity is a risk for asthma and wheeze but not airway hyperresponsiveness. Thorax. 2001;56:4-8.
49. Aaron SD, Vandemheen KL, Boulet LP, et al. Overdiagnosis of asthma in obese and nonobese adults. CMAJ. 2008;179(11):1121-1131.

50. McLachlan CR, Poulton R, Car G, et al. Adiposity, asthma, and airway inflammation. J Allergy Clin Immunol. 2007;119:634-639.

51. Aaron SD, Fergusson D, Dent R, et al. Effect of weight reduction on respiratory function and airway reactivity in obese women. Chest. 2004; 125:2046-2052.

52. Maniscalco M, Zedda A, Faraone S, et al. Weight loss and asthma control in severely obese asthmatic females. Respir Med. 2008;102:102-108.

53. Tantisira KG, Litonjua AA, Weiss ST, et al; for Childhood Asthma Management Program Research Group. Association of body mass with pulmonary function in the Childhood Asthma Management Program (CAMP). Thorax. 2003;58:1036-1041.

54. Rodrigo GJ, Plaza V. Body mass index and response to emergency department treatment in adults with severe asthma exacerbations: a Prospective Cohort Study. Chest. 2007;132:1513-1519.

55. King GG, Brown NJ, Diba C, et al. The effects of body weight on airway calibre. Eur Respir J. 2005;25:896-901.

56. Sin DD, Sutherland ER. Obesity and the lung: 4. Obesity and asthma. Thorax. 2008;63:1018-1023.

57. Shore SA, Schwartzman IN, Mellema MS, et al. Effect of leptin on allergic airway responses in mice. J Allergy Clin Immunol. 2005;115: 103-109.

58. Peters-Golden M, Swern A, Bird SS, et al. Influence of body mass index on the response to asthma controller agents. Eur Respir J. 2006;27: 495-503.

59. Global initiative for chronic obstructive lung disease. Global Strategy for the diagnosis, management, and prevention of chronic obstructive pulmonary disease. Executive Summary. Available from: http://www. goldcopd.org/Guidelineitem.asp?11=2\&12=1\&intId=2180. Updated 2009. Accessed 2010 Jul 15.

60. McNicholas WT. Chronic obstructive pulmonary disease and obstructive sleep apnea. Am J Respir Crit Care Med. 2009;180:692-700.

61. Steuten LM, Creutzberg EC, Vrijhoef HJ, Wouters EF. COPD as a multicomponent disease: inventory of dyspnoea, underweight, obesity and fat free mass depletion in primary care. Prim Care Respir J. 2006; 15:84-91.

62. Eisner MD, Blanc PD, Sidney S, et al. Body composition and functional limitation in COPD. Respir Res. 2007;8(1):7.

63. Magnusson L, Spahn DR. New concepts of atelectasis during general anaesthesia. Br J Anaesth. 2003;91:61-72.

64. Langeron O, Masso E, Huraux C, et al. Prediction of difficult mask ventilation. Anesthesiology. 2000;92:1229-1236.

65. Kheterpal S, Han R, Tremper KK, et al. Incidence and predictors of difficult and impossible mask ventilation. Anesthesiology. 2006; 105:885-891.

66. ASA Task Force on Perioperative Management of Patients with OSA. Practise guidelines for the perioperative management of patients with obstructive sleep apnea. Anesthesiology. 2006;104:1081-1093.

67. Joris JL, Sottiaux TM, Chiche JD, Desaive CJ, Lamy ML. Effect of bi-level positive airway pressure (BiPAP) nasal ventilation on the postoperative pulmonary restrictive syndrome in obese patients undergoing gastroplasty. Chest. 1997;111:665-670.

International Journal of General Medicine

\section{Publish your work in this journal}

The International Journal of General Medicine is an international, peer-reviewed open-access journal that focuses on general and internal medicine, pathogenesis, epidemiology, diagnosis, monitoring and treatment protocols. The journal is characterized by the rapid reporting of reviews, original research and clinical studies across all disease areas.

A key focus is the elucidation of disease processes and management protocols resulting in improved outcomes for the patient.The manuscript management system is completely online and includes a very quick and fair peer-review system. Visit http://www.dovepress.com/ testimonials.php to read real quotes from published authors. 